\title{
Bildgebender prospektiver Marker für Neuroinflammation
}

Fragestellung: Ziel dieser Studie war es, den Vorhersagewert einer pathologischen BHS-Permeabilität für die Konversion von einer Retrobulbärneuritis (RBN) zu einer Multiplen Sklerose (MS) über einen Zeitraum von zwei Jahren zu untersuchen. Zusätzlich wurden Korrelationen zu inflammatorischen Markern im Liquor, Zellmigration und BHS-Dysfunktion vorgenommen.

Hintergrund: Der derzeit beste Prädiktor für die Konversion von einem klinisch isolierten Syndrom (KIS) zur MS gemäß den 2010 revidierten McDonald-Kriterien ist die Anzahl der T2-Läsionen im Schädel-MRT (cMRT) bei Erstmanifestation. Vorarbeiten konnten pathologische Permeabilitäten der Blut-HirnSchranke (BHS) im nicht läsionellen Marklager (normal-appearing white matter; NAWM) von Patienten mit MS nachweisen.

Methoden: 39 Patienten mit einer nicht mehr als 28 Tage zurückliegenden monosymptomatischen RBN im Sinne einer Erstmanifestation erhielten ein 3-Tesla cMRT zur diagnostischen Routine und eine diagnostische Liquorpunktion einschließlich der Bestimmung
Cramer SP, Modvig S, Simonsen $\mathrm{HJ}$ et al. Permeability of the blood-brain barrier predicts conversion from optic neuritis to multiple sclerosis. Brain 2015; 138: $2571-83$ von CXCL10, CLCX13 und MMP9; zusätzlich wurde mittels dynamischer kontrastmittelverstärkter MRT (DCE-MRT) die BHS-Permeabiliät im NAWM und im Thalamus bestimmt. 18 ge- sunde Kontrollpersonen (HC) wurden zum Vergleich eingeschlossen. Informationen bezüglich einer MS-Konversion innerhalb von zwei Jahren nach RBN-Erstmanifestation wurden aus den Krankenakten extrahiert.

Ergebnisse: Die RBN-Patienten hatten verglichen mit den $\mathrm{HC}$ signifikant höhere BHS-Permeabilitäten der NAWM, nicht aber thalamisch. Jene RBN-Patienten, die innerhalb von zwei Jahren eine MS entwickelten ( $\mathrm{n}=17 ; 44 \%)$, hatten signifikant höhere BHS-Permeabilitäten als stabile RBN-Patienten. Logistische Regressionsanalysen zeigten, dass der Nachweis einer pathologischen BHS-Permeabilität (>0,1 ml/100 g/min) das Risiko zur MSKonversion um das 8,5-Fache erhöhte; der Nachweis von $\geq 9$ T2Läsionen im cMRT bei Erkrankungsbeginn war mit einem 53fachen Konversionsrisiko vergesellschaftet. Die Kombination dieser beiden Marker erhöhte die Vorhersagekraft noch zusätzlich. Es bestand eine signifikante Korrelation zwischen der BHS-Permeabilität und der Leukozytenzahl in Liquor sowie den neuroinflammatorischen Markern CXCL10 und MMP9 im Liquor, nicht jedoch zu der Anzahl der T2-Läsionen oder der T2-Läsionslast.

Schlussfolgerungen: Diese Ergebnisse legen nahe, dass die mittels MRT bestimmte BHS-Permeabilität ein neuartiger bildgebender Marker für die Neuroinflammation bei MS ist und zu einem gewissen Ausmaß die zelluläre Durchlässigkeit der BHS reflektiert, während die T2-Läsionslast eher die Länge der subklinischen Phase vor einem Schub darstellt.

\section{- Kommentar von Til Menge, Düsseldorf}

\section{Interessanter MRT-Marker, aber wahrscheinlich nicht alltagstauglich}

Hier wird prospektiv eine 2012 erstmals vorgestellte neuartige Methode angewandt, mittels MRT die (pathologische) Permeabilität der Blut-Hirn-Schranke (BHS) zu messen. Wohlgemerkt handelt es sich nicht um den gut etablierten Nachweis pathologischer Kontrastmittelaufnahme in T2-Läsionen, sondern um den Übertritt kleinster Mengen Gadolinium in das unauffällig erscheinende Marklager und die thalamischen Kerngebiete. Die Daten legen nahe, dass es sich pathophysiologisch um eine Vorstufe der entzündlichen Läsionsentstehung handelt. Somit kann der Nachweis einer BHS-Permeabilität als Risikofaktor für eine Krankheitsaktivität gelten, die die Konversion vom KIS zur MS anzeigt. Der Effekt ist allerdings lange nicht so groß wie der Nachweis von T2-Läsionen im initialen MRT. Leider geht aus der Publikation nicht klar hervor, ob und zu welcher Zeit die Patienten einen Steroidpuls erhalten haben; außerdem begannen $58 \%$ der Patienten zwei bis sechs Wochen nach RBN eine immunmodulatorische Therapie. Ob dieser MRT-Marker Eingang in die klinische beziehungsweise neuroradiologische Rou- tine finden wird, muss bezweifelt werden. Die MRT-Sequenz nämlich dauert zusätzliche 16 Minuten. Für Studienzwecke, beispielsweise zur Stratifizierung von Studienpopulationen oder zum paraklinischen Wirksamkeitsnachweis neuer Substanzen, erscheint der Nachweis einer BHS-Permeabilität mittels MRT - nach Validierung dieser Ergebnisse - durchaus interessant.

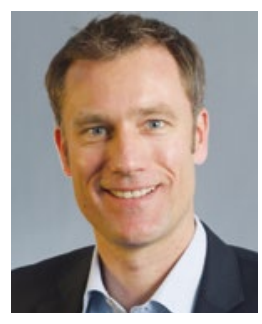

PD Dr. med. Til Menge, Düsseldorf

Oberarzt am Zentrum für Neurologie und Neuropsychiatrie; LVR-Klinikum Düsseldorf E-Mail:menge@uni-duesseldorf.de 